\title{
A Spectral Color Imaging System for Estimating Spectral Reflectance of Paint
}

\author{
Vladimir Bochko \\ Department of Information Technology, Lappeenranta University of Technology, \\ P.O.Box 20, 53851 Lappeenranta, Finland (botchko@lut.fi)
}

\author{
Norimichi Tsumura and Yoichi Miyake \\ Department of Information and Image Sciences, Chiba University, 1-33 Yayoi-cho, \\ Inage-ku, Chiba 263-8522, Japan (tsumura@faculty.chiba-u.jp, \\ miyake@faculty.chiba-u.jp )
}

In this paper, the analysis methods used for developing imaging systems estimating the spectral reflectance are considered. The system incorporates the estimation technique for the spectral reflectance. Several traditional and machine learning estimation techniques are compared for this purpose. The accuracy of spectral estimation with this system and each estimation technique is evaluated and the system's performance is presented.

\section{Introduction}

In this paper, the analysis methods used for developing imaging systems estimating the spectral reflectance are considered. The estimation of the spectral reflectance determines a performance of a high quality color imaging system which is required in digital archives, network museums, e-commerce and telemedicine. ${ }^{1}$ Especially the design of a system for accurate digital archiving of fine art paintings has awakened 
increasing interest. In such a system the digital image is easily examined by using a broadband network. The visitors of museums, art experts and artists would be able appreciate a variety of paintings at any viewing site wherever those paintings are located. In addition, archiving the current condition of a painting with high accuracy in digital form is important to preserve it for the future. Several research groups worldwide have been working on these problems. ${ }^{2,3,4,5,6,7,8,9,10,11,12,13,14}$

Conventional color imaging systems have a limitation that is a dependence of images on the illuminant and characteristics of the imaging system. The imaging systems based on spectral reflectance, unlike the conventional systems, are deviceindependent and capable of reproducing the image of the scene in any illumination conditions. Also, these systems can incorporate the color appearance characteristics of the human visual system. Owing to the fact that spectral characteristics are smoothed, the high-dimensional spectral reflectance is accurately represented by a small number of channel images. ${ }^{15,16,17}$ Therefore, the task of spectral estimation includes statistical analysis of the reflectance spectra and minimization of the estimation error. The choice of error measures is a general topic of broader interest and sometimes contrary in impact. In the archival, ramifications for optimizing more for RMSE versus color difference depend on applications. For example, spectral optimization may better enable the identification of colorants used while color difference optimization may yield superior visual reproductions.

The traditional techniques used for the estimation involve matrix-vector computation and usually assume a linear model of the data. Although the approach based on linear algebra and a nonlinear data model is proposed in the literature, ${ }^{4}$ machine learning 
techniques seem appealing. They estimate spectra of the scene, incorporate the data nonlinearity and involve the training and prediction procedures. Therefore, the neural networks based methods for spectral reconstruction are proposed by Ribes et al. ${ }^{18}$ The tested methods are superior to the pseudo-inverse based estimation method with a quantization noise. Without noise the traditional methods predict better than the neural network because of the highly linear relationship between spectral sets used for training and prediction. To provide color constancy a Bayesian approach of the estimation method is proposed by Brainard and Freeman. ${ }^{19}$ Since the Bayesian approach is computationally demanding, the submanifold method for spectral reflectance estimation that is an intermediate solution between the Bayesian approach and linear estimation methods is described by DiCarlo and Wandell. ${ }^{20}$ The method extends the linear methods and introduces the additional term incorporating the nonlinearity of the data. The method uses a piece-wise linear way to represent the nonlinear data structure and reduces the error value $12 \%$ in comparison with a linear method. It is important that the method particularly reduces large linear errors. The limitation of the method is that it needs a large training set and is insufficient when the data structure is a one-to-many mapping. The properties of the methods considered in this paper are quite close to the submanifold approach ${ }^{20}$ and one of the learning algorithms based on Wiener estimation also gives a piece-wise linear solution.

Recently, many advanced machine learning techniques using neural networks and support vector machines have been introduced and combined in the libraries that are convenient for the purpose. For example, building the estimation methods using the ready-made machine learning algorithms one can get theoretically founded algorithms, 
a unified workflow for a current and future study, and a rich set of methods that provide flexibility for application-oriented research. In this paper, the neural networks algorithms from the Netlab library ${ }^{21,22}$ will be used. They include regression, clustering and pattern recognition methods. Many of these methods are density models based on a likelihood that is important for recognition and convenient for comparison with other methods.

In this study, we statistically analyze the reflectance spectra of color-patch sets of oil and watercolor paintings without noise characteristics, develop three machinelearning based methods and compare them with three traditional methods with a synthetic data set and the real color-patch sets, as well. The traditional methods are linear estimators based on low-dimensional principal component analysis (PCA) approximation and Wiener estimation, and a nonlinear estimator based on multiple regression approximation. The machine learning methods extend the traditional methods for estimating a nonlinear data structure. They include two nonlinear methods based on nonlinear principal component analysis and regression analysis and the method using piece-wise linear Wiener estimation. The method utilizing nonlinear PCA and the method exploiting piece-wise linear Wiener estimation are novel methods. To develop an imaging system, two measures are used for estimation accuracy: spectral color difference (RMSE) and colorimetric color difference (CIE $\left.\Delta \mathrm{E}_{94}\right)$. The former is better for archiving the spectral reflectance and the latter is better for evaluating the appearance of the art paintings under a specific illumination to human observers. 
The paper is arranged as follows: In the following section, we formulate the generalized reconstruction of spectral reflectance from a multichannel image in imaging systems with a reduced number of channels. Next, we describe three traditional methods and three machine learning methods. Then we present the results of the statistical analysis of the reflectance spectra of the color patches. Later on, an experiment with synthetic data and the reflectance spectra of the color patches is described. Finally, the experimental results are discussed and concluding remarks are presented.

\section{Formulation of the Spectral Reflection Estimation}

Fig. 1 shows the image acquisition system. The system consists of the single chip high quality CCD camera and the rotating color wheel comprising several color filters. The response $v$ at position $(x, y)$ of the CCD camera with the $i^{\text {th }}$ color filter is expressed as follows ${ }^{3}$ :

$$
v_{i}(x, y)=\int t_{i}(\lambda) E(\lambda) S(\lambda) r(x, y, \lambda) d \lambda+n_{i}(x, y), \quad i=1, \ldots, m
$$

where $t_{i}(\lambda), E(\lambda), S(\lambda)$ and $r(x, y, \lambda)$ are the spectral transmittance of the $i^{\text {th }}$ filter, the spectral radiance of the illuminant, the spectral sensitivity of the camera, and the spectral reflectance of a painting, respectively. $n_{i}(x, y)$ denotes additive noise in the $i^{\text {th }}$ channel image and $m$ denotes the total number of channels. 


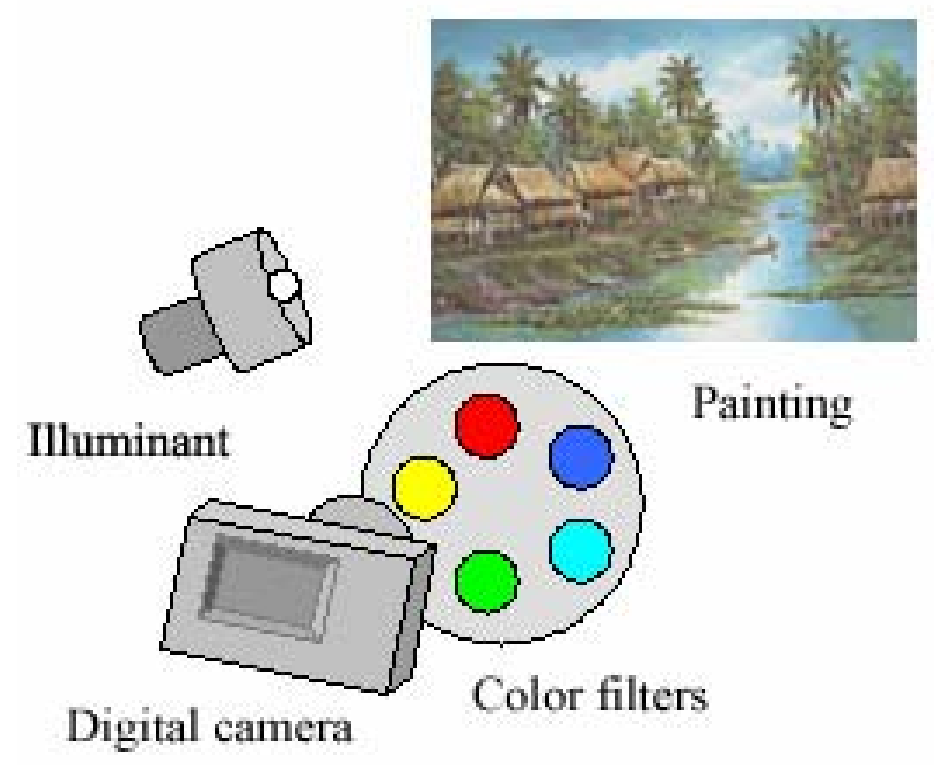

Fig. 1. The image acquisition system.

For mathematical convenience, each spectral characteristic with $l$ wavelengths is expressed as a vector or a matrix. Using vector-matrix notation, we can express Eq. (1) as follows:

$$
\mathbf{v}(x, y)=\mathbf{T}^{T} \mathbf{E S} \quad \mathbf{r}(x, y)+\mathbf{n}(x, y),
$$

where $T$ denotes a transposition, $\mathbf{v}$ is an $m \times 1$ column vector representing the camera response, $\mathbf{r}$ is an $l \times 1$ column vector representing the spectral reflectance of the painting, $\mathbf{T}=\left|\mathbf{t}_{1}, \mathbf{t}_{2}, \ldots, \mathbf{t}_{m}\right|$ is an $l \times m$ matrix in which each column $\mathbf{t}_{i}$ represents the transmittance of the $i^{\text {th }}$ filter, and $\mathbf{E}, \mathbf{S}$ are the $l \times l$ matrices that correspond to the spectral radiance of the illuminant and the spectral sensitivity of the CCD camera, respectively.

Further for the sake of simplicity, $(x, y)$ from $\mathbf{v}, \mathbf{r}$ and $\mathbf{n}$ are omitted. Eq. (2) is rewritten as an overall, linear system matrix $\mathbf{F}=\mathbf{T}^{T} \mathbf{E S}$ with $m \times l$ elements:

$$
\mathbf{v}=\mathbf{F r}+\mathbf{n} .
$$


The response of the spectral CCD camera $\mathbf{v}$ without a noise term is as follows:

$$
\mathbf{v}=\mathbf{F r}
$$

We will call the space spanned by $\mathbf{r}$ a spectral space and the space spanned by $\mathbf{v}$ a sensor space or subspace. The estimation of reflectance spectra is obtained as follows:

$$
\hat{\mathbf{r}}=\mathbf{G v}
$$

where $\mathbf{G}$ is a matrix depending on the estimation method used. In the next sections, six estimation methods are considered.

\section{Traditional Estimation Techniques}

Three approaches are usually used for spectral sensor design. The estimation techniques of reflectance spectra include: the method based on PCA (low-dimensional approximation) (PCE), the method based on Wiener estimation (WE) and the method using multiple regression approximation (MRE). ${ }^{4}$

\section{The Method Based on PCA}

Using spectral reflectance of the training set $\mathbf{r}$ a covariance matrix is computed as follows:

$$
\mathbf{C}=E\left((\mathbf{r}-E(\mathbf{r}))(\mathbf{r}-E(\mathbf{r}))^{T}\right),
$$

where $E()$ is an expectation operator.

An eigendecompisition of the covariance matrix $\mathbf{C}$ determines the matrix $\mathbf{B}=\left|b_{1}, b_{2}, \ldots, b_{k}\right|$, the columns of which are $k$ eigenvectors corresponding to the first $k$ largest eigenvalues. The spectral reflectance is approximated as follows:

$$
\mathbf{r} \cong \mathbf{B w}
$$

where $\mathbf{w}$ is a vector of PCs, $\mathbf{w}=\left|w_{1}, w_{2}, \ldots, w_{k}\right|^{T}$ and $k \leq m$. 
The spectral camera response given by Eq. 4 can be presented by another expression as follows ${ }^{17}$ :

$$
\mathbf{v}=\mathbf{F B w}
$$

The principal components (PCs) are determined as follows:

$$
\mathbf{w}=(\mathbf{F B})^{-1} \mathbf{v} .
$$

Using Eq. 7 and Eq. 9 the estimation matrix $\mathbf{G}$ is as follows:

$$
\mathbf{G}=\mathbf{B}(\mathbf{F B})^{-1}
$$

The estimate of the spectral reflectance of the painting is as follows:

$$
\hat{\mathbf{r}}=\mathbf{G v}=\mathbf{B}(\mathbf{F B})^{-1} \mathbf{v},
$$

where the data is centered by $\mathbf{v} \leftarrow \mathbf{v}-E(\mathbf{F r})$ and $\leftarrow$ means that the expression on the right is calculated and replaces the expression on the left. Finally, the mean value is added as follows:

$$
\hat{\mathbf{r}}=\hat{\mathbf{r}}+E(\mathbf{r})
$$

Better accuracy of estimation can be obtained with Wiener estimation, which is considered next.

\section{The Method Using Wiener Estimation}

The Wiener estimation method minimizes the overall average of the square error between the original and estimated spectral reflectance. ${ }^{3}$ For this method, the correlation matrices $\mathbf{R}_{r r}$ of painting spectra and noise $\mathbf{R}_{n n}$ are first computed, and consequently, the estimation matrix is the following ${ }^{3}$ :

$$
\mathbf{G}=\mathbf{R}_{r r} \mathbf{F}^{T}\left(\mathbf{F} \mathbf{R}_{r r} \mathbf{F}^{T}+\mathbf{R}_{n n}\right)^{-1}
$$

The estimate is as follows:

$$
\hat{\mathbf{r}}=\mathbf{G v}=\mathbf{R}_{r r} \mathbf{F}^{T}\left(\mathbf{F R}_{r r} \mathbf{F}^{T}+\mathbf{R}_{n n}\right)^{-1} \mathbf{v}
$$


If noise is not considered, the estimation matrix is as follows ${ }^{3}$ :

$$
\mathbf{G}=\mathbf{R}_{r r} \mathbf{F}^{T}\left(\mathbf{F R}_{r r} \mathbf{F}^{T}\right)^{-1}
$$

And the estimate is as follows:

$$
\hat{\mathbf{r}}=\mathbf{G v}=\mathbf{R}_{r r} \mathbf{F}^{T}\left(\mathbf{F R}_{r r} \mathbf{F}^{T}\right)^{-1} \mathbf{v}
$$

In this study, the Wiener estimation without consideration to noise is used. The Wiener estimation gives good accuracy for linear data. If the data is nonlinear, the technique based on multiple regression analysis is used.

\section{The Method Using Multiple Regression Analysis}

In the case of nonlinear data, multiple regression analysis gives better results than Wiener estimation. ${ }^{4}$

In the MRE method, the extended data matrix $\mathbf{V}$ of painting spectra is first defined through the data components and their extended set of higher-order terms as follows ${ }^{4}$ :

$$
\mathbf{V}=\mid v_{1}, \ldots, v_{m}, v_{1} \times v_{1}, v_{1} \times v_{2} \ldots, \text { higher }- \text { order terms }, \ldots \mid \text {, }
$$

where $\times$ denotes element-wise multiplication.

Then the estimation matrix is given as follows:

$$
\mathbf{G}=\mathbf{R} \mathbf{V}^{T}\left(\mathbf{V} \mathbf{V}^{T}\right)^{-1},
$$

where $\mathbf{R}$ is a matrix, the columns of which are presented by $n$ spectral samples given by

$$
\mathbf{R}=\left|\begin{array}{llll}
\mathbf{r}_{1}, & \mathbf{r}_{2}, & \ldots, & \mathbf{r}_{n}
\end{array}\right|,
$$

According to the literature ${ }^{4}$, the estimation matrix $\mathbf{G}$ used in MRE is equal to the noiseless variant of the Wiener estimation matrix.

Finally, 


$$
\hat{\mathbf{r}}=\mathbf{G V}=\mathbf{R} \mathbf{V}^{T}\left(\mathbf{V} \mathbf{V}^{T}\right)^{-1} \mathbf{V}
$$

Owing to the fact that new advanced machine learning algorithms are especially relevant for working with a nonlinear structure of data, the machine learning techniques are next discussed for spectral estimation.

\section{Machine Learning Estimation Techniques}

Drawing analogy with the traditional estimation methods, three machine learning techniques are proposed. They include the method based on regressive (nonlinear) PCA (RPCE), the method based on piece-wise linear Wiener estimation (PLWE) and the method using regression analysis (RE). Eq.1 - Eq.5 are valid for all machine learning methods.

\section{The Method Based on Regressive PCA}

The spectral camera response is computed in the following way:

$$
\mathbf{v}=\mathbf{F B f}\left(\mathbf{w}, \boldsymbol{\theta}_{f}\right)
$$

where $\mathbf{f}()$ is a nonlinear vector-valued mapping function and $\boldsymbol{\theta}_{f}$ is a parametric vector.

Then, PCs are defined by the following equation

$$
\mathbf{w}=\mathbf{h}\left((\mathbf{F B})^{-1} \mathbf{v}, \boldsymbol{\theta}_{h}\right),
$$

where $\mathbf{h}()$ is an inverse function, $\mathbf{h}()=\mathbf{f}()^{-1}, \boldsymbol{\theta}_{h}$ is a parametric vector and $\mathbf{v} \leftarrow \mathbf{v}-E(\mathbf{F r})$

The mapping function $\mathbf{h}()$ and parametric vector $\boldsymbol{\theta}_{h}$ are computed using a machine learning algorithm for regression. ${ }^{21}$ In consequence, the spectral estimate of the painting is as follows: 


$$
\hat{\mathbf{r}}=\mathbf{B h}\left((\mathbf{F B})^{-1} \mathbf{v}, \boldsymbol{\theta}_{h}\right)
$$

Finally, the mean value is added as follows:

$$
\hat{\mathbf{r}} \leftarrow \hat{\mathbf{r}}+E(\mathbf{r})
$$

In practice, this method involves a low-dimensional subspace and a higherdimensional subspace including the low-dimensional subspace. For the lowdimensional subspace, where $\mathbf{w}^{(k)}=\left|w_{1}, w_{2}, \ldots, w_{k}\right|^{T}$, the mapping is as follows:

$$
\mathbf{w}^{(k)}=\mathbf{h}\left((\mathbf{F B})^{-1} \mathbf{v}, \boldsymbol{\theta}_{h}\right)=(\mathbf{F B})^{-1} \mathbf{v}
$$

where $\mathbf{v} \leftarrow \mathbf{F r}-E(\mathbf{F r})$

For the higher-dimensional subspace, where

$$
\mathbf{w}^{(p)}=\left|\mathbf{w}^{(k)}, \mathbf{w}^{(k+1: p)}\right|^{T}=\left|w_{1}, w_{2}, \ldots, w_{k}, w_{k+1}, \ldots, w_{p}\right|^{T},
$$

the mapping is done for the higher-order (or weak) PCs as follows:

$$
\mathbf{w}^{(k+1: p)}=\mathbf{h}\left((\mathbf{F B})^{-1} \mathbf{v}, \boldsymbol{\theta}_{h}\right)=\mathbf{h}\left(\mathbf{w}^{(k)}, \boldsymbol{\theta}\right) .
$$

Thus the method uses the low-order real PCs and the higher-order approximated PCs.

\section{The Method Using Piece-Wise Linear Wiener Estimation}

In this section, the other machine learning algorithm for piece-wise linear Wiener estimation is discussed. The main idea of the method is to separate the data structure into parts which are suitable for linear approximation and each part is then estimated by using the linear Wiener estimation method.

For data separation, the clustering algorithm is first required. The data is divided into several clusters $\mathbf{v}_{i}$ using the Gaussian mixture model $(\mathrm{GMM})^{21}$ in a sensor space where $i$ is an index of the cluster. Then for the data of each cluster Wiener estimation is utilized. Using the labels of the data it is easy to compute the cluster covariance 
matrix in the spectral domain needed for estimation. When the $i^{\text {th }}$ cluster covariance matrix $\mathbf{C}_{i}$ of painting spectra is known, the spectral estimate for the $i^{\text {th }}$ cluster is as follows:

$$
\hat{\mathbf{r}}_{i}=\mathbf{G}_{i} \mathbf{v}_{i}=\mathbf{C}_{i} \mathbf{F}^{T}\left(\mathbf{F C}_{i} \mathbf{F}^{T}\right)^{-1} \mathbf{v}_{i}
$$

where $\mathbf{v}_{i} \leftarrow \mathbf{v}_{i}-E\left(\mathbf{F r}_{i}\right)$.

Finally, the mean value is added as follows:

$$
\hat{\mathbf{r}}_{i} \leftarrow \hat{\mathbf{r}}_{i}+E\left(\mathbf{r}_{i}\right)
$$

The estimation procedure is sequentially repeated for all clusters.

\section{The Method Using Regression Analysis}

The estimation method based on the regression analysis is similar to the multiple regression approach. The difference is that nonlinear mapping is used instead of linear mapping and the higher-order terms are not synthesized. For regression analysis based on machine learning the estimate is given as follows:

$$
\hat{\mathbf{r}}=\mathbf{g}(\mathbf{v}, \boldsymbol{\theta})
$$

where $\mathbf{g}$ is a nonlinear vector-valued mapping function and $\boldsymbol{\theta}$ is a vector of parameters.

Then, an $i^{\text {th }}$ entry is defined as follows:

$$
\hat{r}_{i}=g_{i}(\mathbf{v}, \boldsymbol{\theta})
$$

There are several regression algorithms ${ }^{21}$ but only the regression method based on the radial basis function $(\mathrm{RBF})$ is used in this study for all methods. The reason is that the RBF method is relatively fast and performs well. 


\section{Additional Techniques}

All machine learning algorithms may need the additional techniques that help in parameter adjustment.

The regressive PCA method used in this study is a technique which combines the PCA and nonlinear regression methods. ${ }^{23}$ In general, the ways utilized in both approaches to detect the underlying dimensionality of the data can be combined. For PCA, this is an analysis of the residual energy depending on a number of PCs. Furthermore, for regression methods this is Automatic Relevance Determination (ARD). ${ }^{21}$ The ARD method defines the statistical dependence between the PCs, and in the case of the dependency between the tested components and a target component the tested components are relevant to approximate the target component. However, this technique will not be used in this study. For the regressive PCA the number of real PCs will be given and a number of approximated PCs will be used as a free parameter.

The piece-wise linear Wiener estimation approach needs to determine the number of linear components for using a clustering procedure. This is done based on the model selection of the mixed distribution. ${ }^{24}$ After that the Gaussian mixture model ${ }^{21}$ with a given number of clusters is used to extract linear components.

\section{Statistical Properties of Reflectance Spectra}

For statistical analysis of the spectral reflectance of paintings we use five sets of color patches of oil or watercolor paint as follows: set A, 336 patches of paint (reflectance of paint); set B, 60 patches of paint (Turner acryl gouache); set C, 60 patches of paint 
(Turner golden acrylics); set D, 91 patches of paint (Kusakabe oil paint) and set E, 18 patches of paint (Kusakabe haiban). All sets were extracted from the standard object color spectral database constructed by the Spectral Characteristic Database Construction Working Group. ${ }^{25}$ These sets have a spectral range of 400-700 nm and samples are evenly taken at $10 \mathrm{~nm}$.

The set A is used for training the algorithms and the sets B-E are used for prediction of the spectral reflectance. Therefore, linear and nonlinear principal component analysis was carried out only for the set A. According to a previous publication ${ }^{3}$, five PCs of linear PCA are good enough for accurate spectral estimation. Hence the spectral set A and its first five PCs that have a residual energy of $0.16 \%$ are analyzed and shown in Fig. 2 and Fig. 3, respectively.

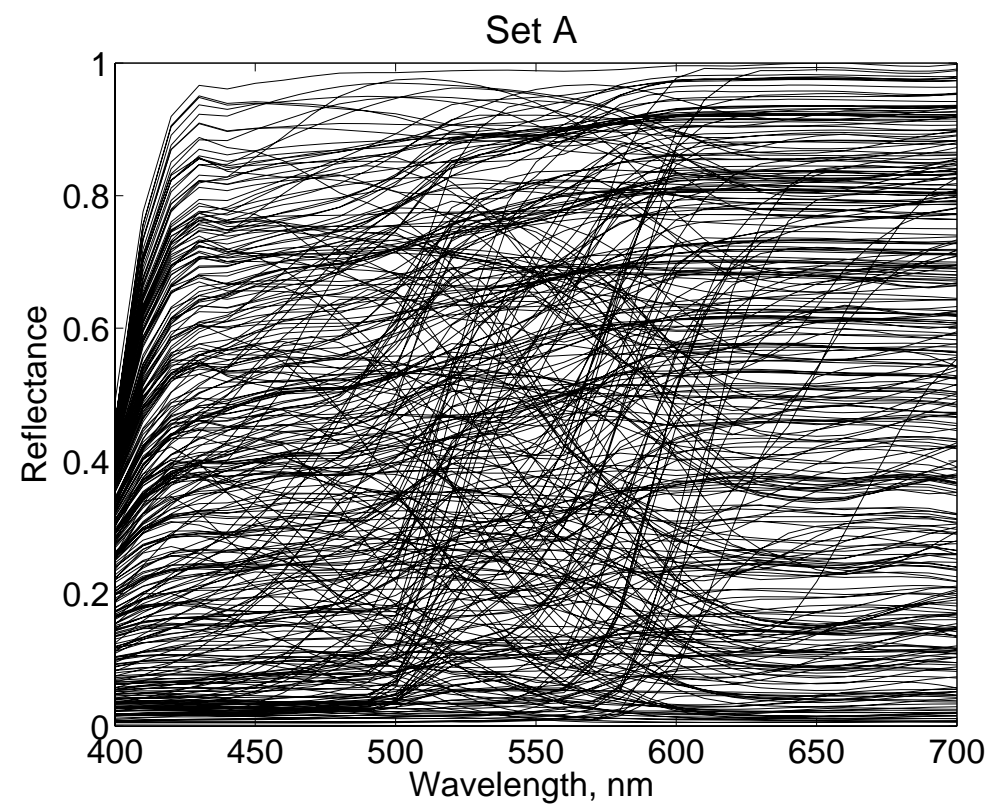

Fig. 2. Reflectance spectra of the set A of paint patches. 

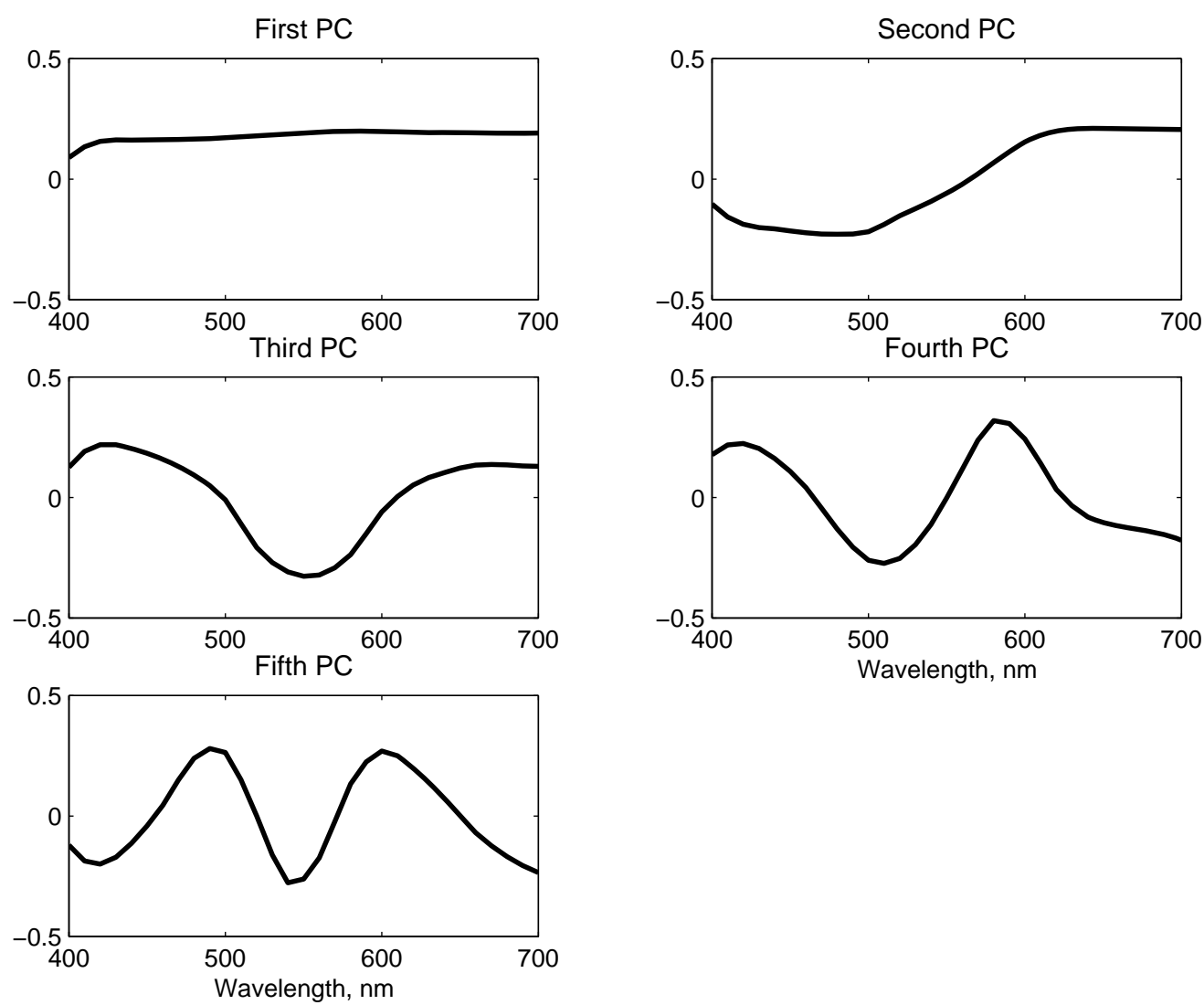

Fig. 3. First five principal components of the set A of paint patches.

If regressive PCA is applied to utilize the five real PCs and several approximated PCs of the set A, the average RMSE value of the spectral approximation is reduced (Fig. 4). This illustrates the fact that there is a way to improve the degree of accuracy for representing spectra by incorporating the nonlinearity of the data.

\section{Experiment}

\section{Synthetic Data}

In this section, the nonlinear dataset is first synthesized and then all methods for spectral estimation are tested with a synthetic set. It is assumed that one channel response is used while the data simulating spectra is two-dimensional. The purpose of the test is to show the feasibility of the method to work with data which has a nonlinear structure. 


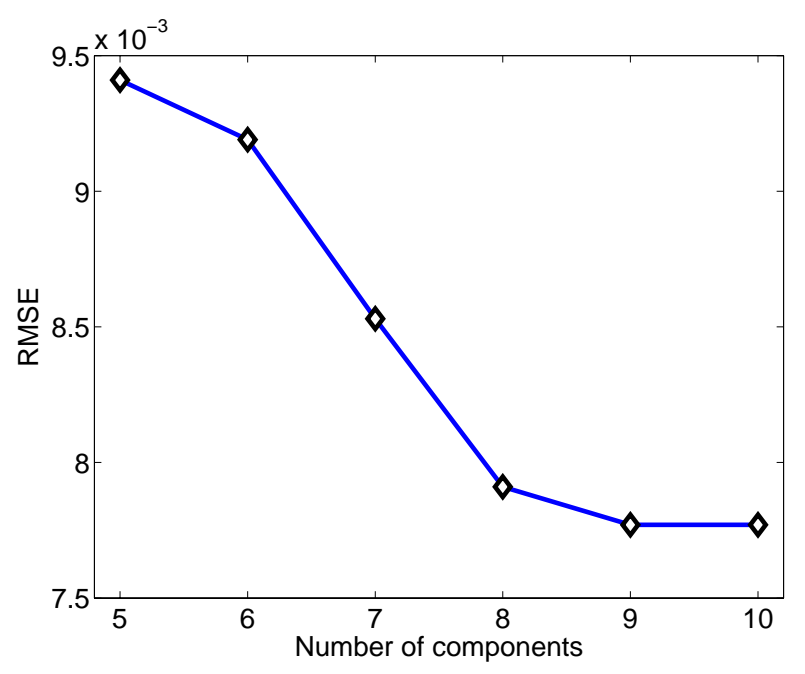

Fig. 4. The average RMSE of spectral approximation for the set A using regressive PCA. The first five components are given by PCA and the components 6-10 are approximated by regressive PCA.

Thus two data components are generated for the test. The first component $x_{1}$ is uniformly distributed in the range $-0.2-0.5$ and another one is $x_{2 i}=\left(x_{1 i}-0.5\right)^{4}$. Finally, a zero-mean Gaussian noise with the standard deviation 0.007 was added to the generated components. The estimation result of the synthetic data is presented in Fig. 5. A vector $\mathbf{F}$, a vector $\mathbf{b}_{1}$, that is a first PCA eigenvector from $\mathbf{B}$ and the curve corresponding to an underlying subspace are shown in Fig. 5. The original (synthesized) data and the estimates for each method are shown by gray dots in Fig. 5.

Although the WE method is superior to the PCE based method, the PCE and WE methods give poor estimates for the data. The MRE, RPCE and PLWE methods are relatively good for estimation. The RE method gives the best result from among these methods. 

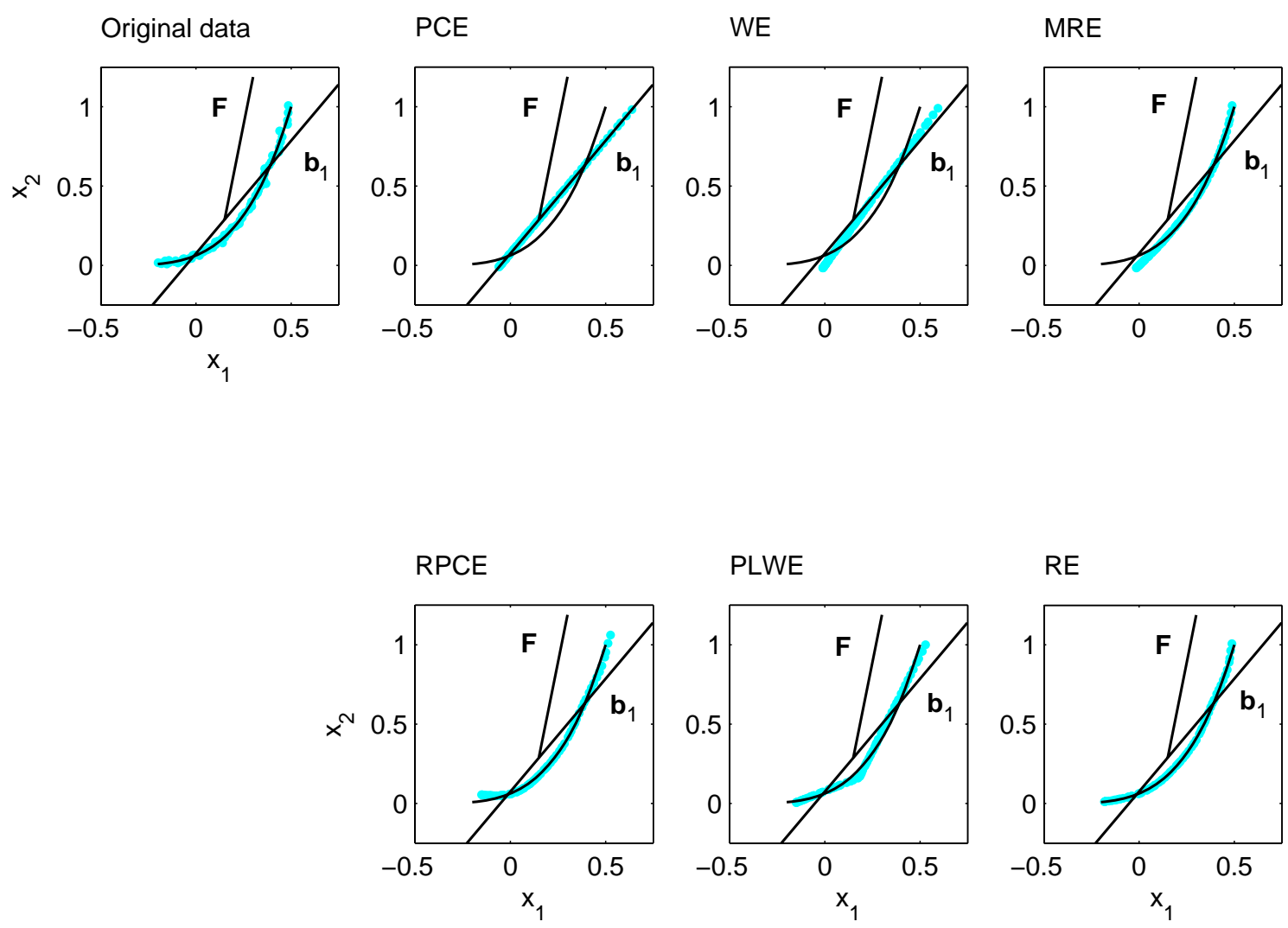

Fig. 5. The estimation results for the synthetic data and different estimation methods.

\section{Real Data}

An experiment was conducted with sets A-E described above. The set A is used for training while the other sets are used for prediction. The spectral transmittance characteristics of the separation filters used in a CCD camera are given in Fig. 6. The spectral sensitivity of a CCD area sensor (Phase One 3072 (horizontal pixels) $\times 2060$ (vertical pixels), 14 bits) is presented in Fig. 7. The illumination source is D65. 


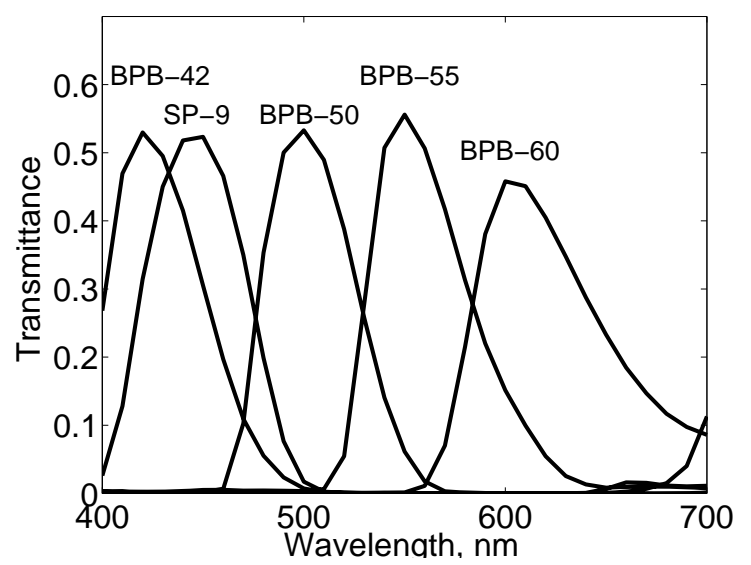

Fig. 6. The spectral transmittance characteristics of the filters.

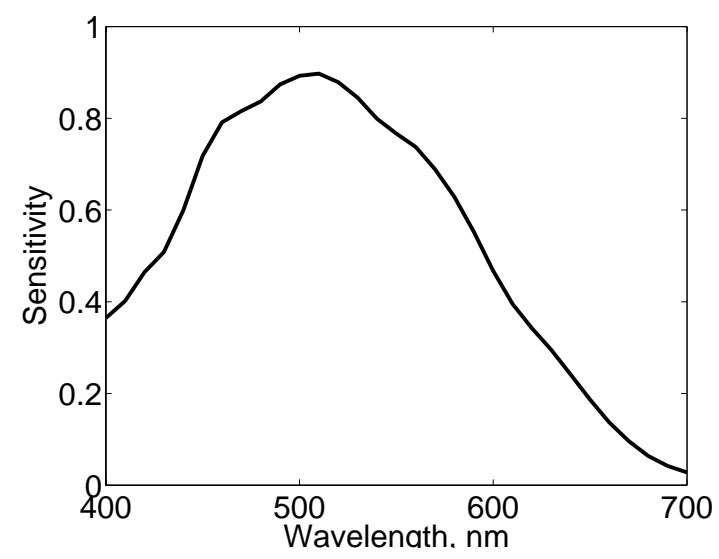

Fig. 7. The spectral sensitivity of the camera.

The parameters used in the test are the following: The five PCs are exploited for PCE and RPCE. In addition, the RPCE approach uses the PCs approximating the real sixth, seventh, eighth and ninth PCs. For the PLWE method a mixture of Gaussian components is used for clustering where the number of components is defined in a test based on the model selection of the mixed distribution. The MRE technique uses the terms beginning with the first-order to the second-order ones. For the RE method, regression is based on the radial basis function using the Gaussian function. 20 neurons and 7 iterations are used in this case. 
A variational Bayesian model selection method for the mixture distribution ${ }^{24}$ in the sensor space defines the number of components for the PLWE method. For this, the program is rerun ten times. The results are presented in Table 1 where the first row shows the test number and the second row shows the number of components determined by the algorithm. Fig. 8 illustrates the variational likelihood bound over the model selection of 336 painting spectra (set A). Initially, the model has ten Gaussians. The vertical lines show the removal of the components from the model. Finally, two components are selected.

Table 1. The number of components for piece-wise linear Wiener estimation

\begin{tabular}{|r|l|l|l|l|l|l|l|l|l|l|}
\hline Test number & 1 & 2 & 3 & 4 & 5 & 6 & 7 & 8 & 9 & 10 \\
\hline Number of components & 2 & 2 & 1 & 1 & 2 & 2 & 2 & 2 & 2 & 2 \\
\hline
\end{tabular}

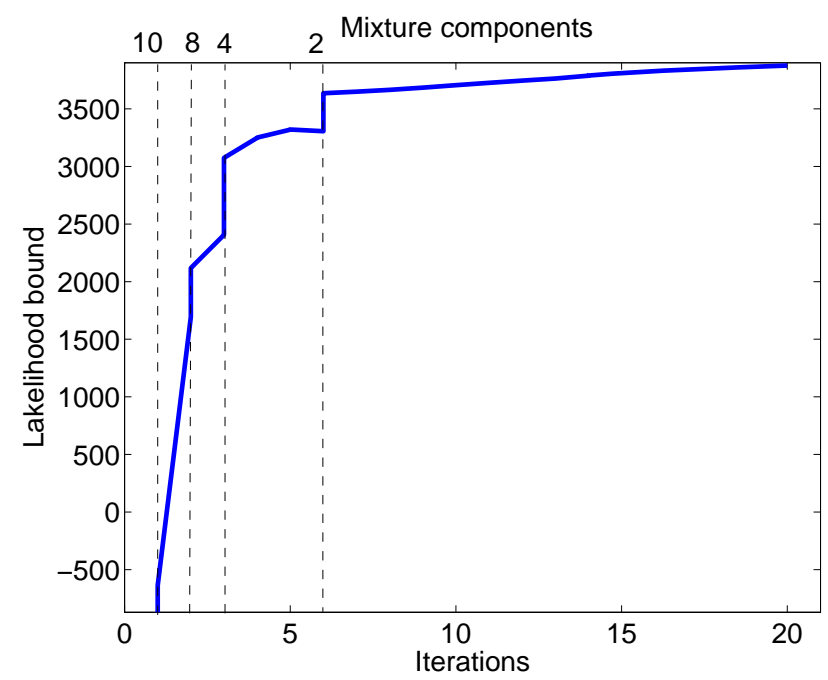

Fig. 8. The variational likelihood bound over the model selection of 336 painting spectra (set A). 
If the estimation values of spectral reflectance are less than zero or greater than one then they are equalized to zero or one, respectively

In Table 2 and Table 3, the average and maximum RMSE values for each set are given for the traditional methods and methods based on machine learning algorithms.

Table 2. The average and maximum (in parentheses) RMSE values for PCE, WE and MRE

\begin{tabular}{|r|l|l|l|}
\hline & PCE & WE & MRE \\
\hline Set A & $0.0516(0.2458)$ & $0.0155(0.1633)$ & $0.0123(0.1159)$ \\
\hline Set B & $0.0836(0.3952)$ & $0.0346(0.1712)$ & $0.0324(0.1732)$ \\
\hline Set C & $0.0889(0.3469)$ & $0.0466(0.2478)$ & $0.0397(0.2158)$ \\
\hline Set D & $0.0917(0.4083)$ & $0.0403(0.2304)$ & $0.0352(0.2075)$ \\
\hline Set E & $0.0917(0.3136)$ & $0.0330(0.1416)$ & $0.0281(0.1199)$ \\
\hline
\end{tabular}

Table 3. The average and maximum (in parentheses) RMSE values for RPCE, PLWE and RE

\begin{tabular}{|r|l|l|l|}
\hline & RPCE & PLWE & RE \\
\hline Set A & $0.0512(0.2447)$ & $0.0142(0.1522)$ & $0.0123(0.1047)$ \\
\hline Set B & $0.0834(0.3928)$ & $0.0343(0.1683)$ & $0.0315(0.1731)$ \\
\hline Set C & $0.0887(0.3452)$ & $0.0450(0.2350)$ & $0.0379(0.2010)$ \\
\hline Set D & $0.0912(0.4066)$ & $0.0376(0.2209)$ & $0.0349(0.1992)$ \\
\hline Set E & $0.0910(0.3122)$ & $0.0339(0.1185)$ & $0.0275(0.1062)$ \\
\hline
\end{tabular}


In Table 4 and Table 5, the average and maximum $\mathrm{CIE} \Delta \mathrm{E}_{94}$ values for each set are given for the traditional methods and methods based on machine learning algorithms.

Table 4. The average and maximum (in parentheses) CIE $\Delta \mathbf{E}_{94}$ values for PCE, WE and MRE

\begin{tabular}{|r|l|l|l|}
\hline & PCE & WE & MRE \\
\hline Set A & $0.72(13.65)$ & $0.17(4.03)$ & $0.15(1.68)$ \\
\hline Set B & $2.96(21.00)$ & $0.58(2.84)$ & $0.54(2.13)$ \\
\hline Set C & $2.36(15.42)$ & $0.80(4.08)$ & $0.59(4.21)$ \\
\hline Set D & $2.43(19.24)$ & $0.71(5.18)$ & $0.55(3.37)$ \\
\hline Set E & $1.32(3.57)$ & $0.37(2.34)$ & $0.31(1.18)$ \\
\hline
\end{tabular}

Table 5. The average and maximum (in parentheses) CIE $\Delta \mathbf{E}_{94}$ values for RPCE, PLWE and RE

\begin{tabular}{|r|l|l|l|}
\hline & RPCE & PLWE & RE \\
\hline Set A & $0.81(14.89)$ & $0.16(3.46)$ & $0.17(3.16)$ \\
\hline Set B & $3.34(23.15)$ & $0.67(2.65)$ & $0.59(2.65)$ \\
\hline Set C & $2.51(14.90)$ & $1.033(8.47)$ & $0.82(3.47)$ \\
\hline Set D & $2.71(20.86)$ & $0.8623(8.19)$ & $0.74(2.92)$ \\
\hline Set E & $1.89(5.14)$ & $0.57(2.00)$ & $0.71(2.79)$ \\
\hline
\end{tabular}

In general, the results presented in Table 2 - Table 5 demonstrate that for the RMSE values the machine learning methods give slightly better results than their traditional opposite methods while the traditional methods have smaller CIE $\Delta \mathrm{E}_{94}$ values. The exception is the RE method which has better prediction in comparison with the other methods for the maximal error of the color difference. 
The methods are also tested using computational time. The CPU time in seconds for the set $\mathrm{A}$ is presented in Table 6 . For the algorithms, the CPU time is given separately for training (upper row) and prediction (lower row). In Table 6, zero values are given for the CPU time, which is very small (this corresponds to several matrix-vector multiplications). Matlab 6.5, the Intel Pentium III Processor, $1066 \mathrm{MHz}$ and $248 \mathrm{MB}$ of RAM are used in the test. The test shows that the traditional methods are faster than the machine learning methods. However, the prediction time for the machine learning methods is relatively short.

Table 6. The CPU time in seconds

\begin{tabular}{|c|c|c|c|c|c|}
\hline PCE & WE & MRE & RPCE & PLWE & RE \\
\hline 0.04 & 0.0 & 0.01 & 0.35 & 0.38 & 6.49 \\
0.0 & 0.0 & 0.01 & 0.03 & 0.22 & 0.18 \\
\hline
\end{tabular}

To see whether any nonlinearity is presented in the estimated spectra we measure the average RMSE value after estimation of spectral reflectance using PCA and RPCA. The results are shown in Table 7 for PCA with the five PCs (upper number) and for RPCA with the five real PCs and five approximated (from six to ten) PCs (lower number). Then, the ratio between these two RMSE values is determined and presented in Table 8.

From Table 8, one can see that the RE and RPCE methods have ratio values close to the original data set. The MRE and PLWE methods give results which are farther from the original data set. The PCE and WE ratio values are the most different from the original data in comparison with the other methods. 
Table 7. The average RMSE value after spectral estimation for PCA with the five PCs (upper number) and for RPCA with the five real components and five approximated components (lower number).

\begin{tabular}{|c|c|c|c|c|c|c|}
\hline Set A & PCE & WE & MRE & RPCE & PLWE & RE \\
\hline 0.00941 & 0.00441 & 0.00043 & 0.00728 & 0.00614 & 0.00453 & 0.00807 \\
0.00772 & 0.00422 & 0.00048 & 0.00539 & 0.00479 & 0.00423 & 0.00626 \\
\hline
\end{tabular}

Table 8. The ratio between the RMSE values for PCA and RPCA

\begin{tabular}{|c|c|c|c|c|c|c|}
\hline Set A & PCE & WE & MRE & RPCE & PLWE & RE \\
\hline 1.21 & 1.04 & 0.88 & 1.35 & 1.28 & 1.07 & 1.29 \\
\hline
\end{tabular}

From among the traditional methods the method based on MRE produces the best result. The method has small RMSE and CIE $\Delta \mathrm{E}_{94}$ values in the training set and sets used for prediction. While the RMSE values for all machine learning methods are slightly better in comparison with the traditional methods, the CIE $\Delta \mathrm{E}_{94}$ values of the methods based on machine learning except the RE method are higher. The overall means of average color differences for the traditional methods are 1.95 (PCE), 0.52 (WE) and 0.42 (MRE) and for the learning methods 2.25 (RPCE), 0.65 (PLWE) and $0.6(\mathrm{RE})$. Thus, the color differences using the machine learning methods are smaller than the differences between the traditional methods. The RE method incorporates nonlinearity of data that is clearly seen from Table 8 . The generalization of the data given by the RE method is very good in comparison with the other methods. This follows from predicting the maximum $\mathrm{CIE} \Delta \mathrm{E}_{94}$ values. However, given the processing and execution times the MRE method gives a better average and in two out of five cases smaller maximum color difference errors than the RE method. Although 
the traditional methods are less time consuming than the machine learning methods, the prediction time for the learning methods is short enough.

In general, the traditional methods look more desirable than the learning methods. This is contrary to the initial result shown in Fig. 5 where the learning methods are superior to the traditional methods. This can be explained as follows. In this study the sensor space (subspace) dimensionality is defined by the five given filters. Although the subspace is not optimal (close to optimal) its dimensionality is rather high. Recently, it was shown that for reflectance spectra the dimensionality of the nonlinear subspace is approximately three. ${ }^{26}$ Thus, one can expect that for spectral imaging systems having the low dimensional sensor space or fewer channels the learning based methods are more efficient. We will consider this problem in a future study.

\section{Conclusions}

We have compared the methods for estimating the spectral reflectance of art paintings for the development of spectral color imaging systems. Three traditional methods and three methods based on machine learning for spectral reflectance estimation of paint were utilized. The traditional methods include two linear methods - the method based on PCA and the method based on Wiener estimation - and one method using multiple regression analysis. We introduced two novel machine learning methods utilizing regressive PCA and piece-wise linear Wiener estimation. Thus, the machine learning methods include two methods working with a global nonlinear data structure - the method based on regressive PCA and the method based on regression analysis - and the method using piece-wise linear Wiener estimation. Similarly to the submanifold method $^{20}$, the learning methods used are between the linear and Bayesian approaches 
and the methods working with nonlinear data have a limitation. They work only with a data structure with a one-to-one mapping. Finally, we synthesized a spectral color imaging system implementing the different estimation methods and demonstrated the possibility for accurately estimating the reflectance spectra using the presented techniques.

\section{Acknowledgments}

The authors thank the Academy of Finland for the funding granted to this study.

\section{References}

${ }^{1}$ Y. Miyake, Evaluation of Image Quality Based on Human Visual Characteristics, in Proc. of the First International Workshop on Image Media Quality and its Applications, Nagoya, Japan, pp. 10-14 (2005).

${ }^{2}$ Y. Miyake, Y. Yokoyama, N. Tsumura, H. Haneishi, K. Miyata, and J. Hayashi, Development of Multiband Color Imaging Systems for Recording of Art Paintings, in Color Imaging: Device-Independent Color, Color Hardcopy, and Graphic Arts, Proc. of SPIE 3648, pp. 218-225 (1999).

${ }^{3}$ H. Haneishi, T. Hasegawa, A. Hosoi, Y. Yokoyama, N. Tsumura and Y. Miyake, System Design for Accurately Estimating the Spectral Reflectance of Art Paintings, Applied Optics 39, (35) pp. 6621-6632 (2000).

${ }^{4}$ N. Tsumura, H. Haneishi, and Y. Miyake, Estimation of Spectral Reflectances from Multi-Band Images by Multiple Regression Analysis, Japanese Journal of Optics, 27, (7) pp. 384-391 [in Japanese] (1998). 
${ }^{5}$ M. J. Vhrel and H. J. Trussel, Color Correction Using Principal Components, Color Res. App. 17, pp. 328-338 (1992).

${ }^{6}$ M. J. Vrhel and H. J. Trussel, Filter Considerations in Color Correction, IEEE Trans. Image Process. 3, pp. 147-161 (1994).

${ }^{7}$ S. Goodall, P H. Lewis, K. Martinez, P. A. S. Sinclair, F. Giorgini, M. J. Addis, M. J. Boniface, C. Lahanier, and J. Stevenson, SCULPTEUR: Multimedia Retrieval for Museums, in Proc. of the International Conference Image and Video Retrieval CIVR 2004, Dublin, Ireland, pp. 638-646 ( 2004).

${ }^{8}$ K. Martinez, J. Cupitt and D. Saunders, High Resolution Colorimetric Imaging of Paintings, in Cameras, Scanners, and Image Acquisition Systems, Proc. of SPIE 1901, pp. 25-36 (1993).

9 J. E. Farrell, J. Cuppitt, D. Saunders and B. A. Wandel, Estimating Spectral Reflectances of Digital Images of Art, in Proc. of the International Symposium of Multispectral Imaging and Color Reproduction for Digital Archives, Chiba, Japan, pp. 58-64 (1999).

10 J. Y. Hardeberg, H. Brettel and F. Schmitt, Spectral Characterization of Electronic Cameras, in Electronic Imaging: Processing, Printing and Publishing in Color, Proc. of SPIE 3409, pp. 100-109 (1998).

${ }^{11}$ H. Maître, F. Schmitt, J.-P. Crettez, Y. Wu and J. Y. Hardeberg, Spectrophotometric Image Analysis of Fine Art Paintings, in Proc. of the Fourth Color Imaging Conference, Scottsdale, Arizona, pp. 50-53 (1996).

12 M. Hauta-Kasari, K. Miyazava, S. Toyooka, J. Parkkinen and T. Jaaskelainen, Spectral Vision System Based on Rewritable Broad Band Color Filters, in Proc. of the 
International Symposium of Multispectral Imaging and Color Reproduction for

Digital Archives, Chiba, Japan, pp. 155-158 (1999).

${ }^{13}$ P. D. Burns, and R. S. Berns, Analysis of Multispectral Image Capture, in Proc. of the Fourth Color Imaging Conference, Scottsdale, Arizona, pp. 19-22 (1996).

${ }^{14}$ F. H. Imai and R. S. Berns, High-resolution Multi-spectral Image Archives: a Hybrid Approach, in Proc. of the Fourth Color Imaging Conference, Scottsdale, Arizona, pp. 224-227 (1998).

${ }^{15}$ L. T. Maloney, Evaluation of Linear Models of Surface Spectral Reflectance with Small Number of Parameters, J. Opt. Soc. Am. A 10, pp.1673-1683 (1986).

16 J. Parkkinen, J. Hallikainen and T. Jaaskelainen, Characteristic Spectra of Munsell Color, J. Opt. Soc. Am. A 6, pp. 318-322 (1989).

${ }^{17}$ M. J. Vrhel, R. Gershon, and L. S. Iwan, Measurement and Analysis of Object Reflectance Spectra, Color Res. App. 19, pp. 4-9 (1994).

18 A. Ribes, F. Schmitt and H. Brettel, Reconstructing Spectral Reflectances of Oil Pigments with Neural Networks, in Proc. of the Third International Conference on Multispectral Color Science, Joensuu, Finland, pp. 9-12 (2001).

19 D. H. Brainard and W. T. Freeman, Bayesian Color Constancy, J. Opt. Soc. Am. A 14, pp. 1393-1411 (1997).

${ }^{20}$ J. M. DiCarlo and B. A. Wandell, Spectral Estimation Theory: Beyond Linear but before Bayesian, J. Opt. Soc. Am. A 20, pp.1261-1270 (2003).

21 I. T. Nabney, Netlab Algorithms for Pattern Recognition (Springer, 2002)

${ }^{22}$ Netlab Toolbox, http://www.ncrg.aston.ac.uk/netlab 
${ }^{23}$ V. Bochko and J. Parkkinen, Principal Component Analysis Using Approximated Principal Components, Research Report 90, Department of Information Technology, Lappeenranta University of Technology, pp. 1-7 (2004).

24 A. Corduneanu, A. and C. M. Bishop, Variational Bayesian Model Selection for Mixture Distributions, in Proc. of the Eighth International Conference on Artificial Intelligence and Statistics, edited by T. Richardson and T. Jaakkola, pp. 27-34 (Morgan Kaufmann, 2001).

25 J. Tajima, M. Tsukada, Y. Miyake, H. Haneishi, N. Tsumura, M. Nakajima, Y. Azuma, T. Iga, M. Inui, N. Ohta, N. Ojima and S. Sanada, Development and Standardization of a Spectral Characteristics Data Base for Evaluating Color Reproduction in Image Input Devices, in Electronic Imaging: Processing, Printing, and Publishing in Color, Proc. of SPIE 3409, pp. 42-50 ( 1998).

${ }^{26}$ B. Funt, D. Kulpinski, and V. Cardei, Non-Linear Embeddings and the Underlying Dimensionality of Reflectance Spectra and Chromaticity Histograms, in Proc. of the Ninth Color Imaging Conference: Color Science, Systems and Applications, pp. 126129 (2001). 